\title{
The need for nutritional assessment and interventions based on the prognostic nutritional index for patients with femoral fractures: a retrospective study
}

\section{Miao He}

Soochow University Medical College

\section{Qinghong Fan}

Soochow University Medical College

\section{Yuhang Zhu}

Affiliated Hospital of Zunyi Medical College

Dexing Liu

Affiliated Hospital of Zunyi Medical College

\section{Xingxing Liu}

Affiliated Hospital of Zunyi Medical College

\section{Shan Xu}

Affiliated Hospital of Zunyi Medical College

Jiachen Peng

Affiliated Hospital of Zunyi Medical College

Zhaoqiong Zhu ( $\nabla$ zhuzhaoqiong@zmu.edu.cn )

Affiliated Hospital of Zunyi Medical College https://orcid.org/0000-0001-5279-2984

\section{Research}

Keywords: prognostic nutritional index, femoral fracture, adverse perioperative outcomes, independent factors, nutrition

Posted Date: April 27th, 2021

DOl: https://doi.org/10.21203/rs.3.rs-463024/v1

License: (c) (i) This work is licensed under a Creative Commons Attribution 4.0 International License. Read Full License 
Version of Record: A version of this preprint was published at Perioperative Medicine on December 1st, 2021. See the published version at https://doi.org/10.1186/s13741-021-00232-1. 


\section{Abstract \\ Background}

The incidence of adverse perioperative outcomes in surgery for femoral fractures is quite high and is associated with malnutrition. This study aimed to identify independent factors and assess the predictive value of the prognostic nutritional index (PNI) for perioperative adverse outcomes in patients with femoral fractures.

\section{Methods}

This retrospective study included 343 patients who underwent surgery for a single femur fracture. Demographic characteristics, surgery and anaesthesia records, and blood test results at admission, 1 day postoperatively, and before discharge were evaluated using logistic regression analysis. The discriminatory ability of the independent factors was assessed using the receiver operating characteristic curve analysis, and DeLong's test was used to compare the area under the curve (AUC).

\section{Results}

Overall, 159 patients (46.4\%) experienced adverse perioperative outcomes. Among these, $123(35.9 \%)$ had lower limb vein thrombus, $68(19.8 \%)$ had hospital-acquired pneumonia, $6(1.7 \%)$ were transferred to the postoperative intensive care unit, $4(1.2 \%)$ had pulmonary embolism, $3(0.9 \%)$ died during hospitalisation, and $9(2.6 \%)$ had other adverse outcomes, including incision disunion, renal and liver function impairment, acute heart failure, acute cerebral infarction, and stress gastroenteritis. The PNI at admission, age, postoperative hospital stay, time to admission, hypertension, combined injures, and surgery type were independent factors for adverse perioperative outcomes. Based on the AUC (PNI at admission: 0.772 (0.723-0.821), $P<0.001$; age: 0.678 (0.622-0.734), $P<0.001$; postoperative hospital stay: 0.608 (0.548$0.668), P=0.001$; time to admission: $0.585(0.525-0.646), P=0.006)$, the PNI at admission had optimal discrimination ability, indicating its superiority over other independent factors (age vs. PNI at admission, $P=0.002$; postoperative hospital stay vs. PNI at admission, $P<0.001$; time to admission vs. PNI at admission, $P<0.001)$.

\section{Conclusions}

Nutritional assessment and appropriate intervention strategies on admission are necessary for patients with femoral fractures, and the PNI at admission may be a good nutritional assessment indicator.

\section{Background}


The number of individuals sustaining fractures is increasing globally, particularly among the elderly population. ${ }^{1,2}$ Clinically, femoral fractures are one of the most encountered fracture types and are associated with higher rates of complications, profound reduction in quality of life, and increase in morbidity, mortality, and economic costs. ${ }^{2-4}$ Femoral fractures are generally classified according to the site of fracture as proximal, shaft, or distal femoral fractures. The incidence of proximal femoral fractures, classified as hip fractures, is the highest and is likely to continue to increase in the future owing to the rapidly ageing population and associated occurrence of osteoporosis. ${ }^{5-7}$ Femoral shaft fractures, which are predominantly noted in young people with healthy bones, ${ }^{8}$ are primarily caused by road traffic accidents (being crushed or run over) or falling from a great height. ${ }^{8}$ Distal femoral fractures are rare injuries, accounting for approximately $2 \%$ of all femoral injuries, ${ }^{9}$ and often develop due to vehicular trauma or sports activities with varus or valgus impact at the knee. ${ }^{9}$

Surgery is usually the best treatment, and it is often performed for patients with femoral fractures. However, the incidence of adverse perioperative outcomes is quite high, including lower limb vein thrombus or pulmonary embolism, pneumonia, incision disunion or infection, acute exacerbation of underlying chronic diseases, transfer to the intensive care unit, and even death. Age, trauma, stress, surgery, anaemia, bleeding, infection, pain, activity limitation, and a bedridden state are commonly considered to be the causes for the aforementioned outcomes. Such patients may be at risk for protein catabolism and malnutrition. Further, nutrition has a major influence on fracture healing, and fracture healing impairment has been observed among malnourished and undernourished individuals. ${ }^{2,10,11}$ Protein-depleted patients with a hip fracture have shown higher complication rates and longer hospitalisation periods. ${ }^{10}$ Notably, Hughes et al. have shown that nutritional improvement leads to increased muscle mass in the leg and greater bone mineral density in the fractured callus in proteinmalnourished rats with femoral fractures. ${ }^{2}$ Nevertheless, only a few studies have investigated the impact of nutrition on adverse outcomes in patients with femoral fractures, specifically during the perioperative stage.

The prognostic nutritional index (PNI), initially proposed by Buzby et al., ${ }^{12}$ is a comprehensive index for evaluating the nutritional status of patients undergoing surgery. ${ }^{13,14}$ Currently, a low PNI, as a proxy for subpar perioperative nutritional status, is reportedly a significant predictor of poor postoperative outcomes and increased mortality in various malignancies. ${ }^{15,16}$ However, studies on PNI focusing on the perioperative adverse outcomes of patients undergoing surgery for femoral fractures are almost nonexistent. Therefore, in this retrospective study, we aimed to determine the independent factors for perioperative adverse outcomes and evaluate the predictive value of the PNI in patients with femoral fractures.

\section{Methods}

\section{Data source}


The data for this retrospective observational study were extracted from the Hospital Information System (TianJian Technology Co., Ltd., Beijing, China) and Anaesthesia Information Management System (Medical System Technology Co., Ltd., Suzhou, Jiangsu, China). The Hospital Information System and the Anaesthesia Information Management System, which maintain a complete record of healthcare services, are electronic medical record management systems for hospitals in China.

\section{Patients}

A retrospective review was performed using data from a database of 446 patients who underwent surgery for a single femur fracture during hospitalisation between January 2018 and December 2018 at the Affiliated Hospital of Zunyi Medical University. These patients did not receive nutritional counselling during hospitalisation. The main nutritional interventions, such as infusion of human serum albumin (ALB), amino acid, and fat emulsion, were used as conventional therapies only when the ALB concentration was $<30 \mathrm{~g} / \mathrm{l}$. The case definition of femur fracture was based on specific diagnosis codes from the International Classification of Diseases, Tenth Revision (ICD-10, S72). These codes were listed as the primary diagnosis on the electronic inpatient healthcare claim submitted to the Hospital Information System. The exclusion criteria were as follows: (i) reoperation or surgeries at multiple sites ( $n$ =44); (ii) incomplete data ( $n=27$ ); (iii) systemic wasting diseases (tuberculosis, tumours, and hyperthyroidism; $n=19)$; (iv) age < 18 years $(n=4)$; (v) history of thromboembolism ( $n=4)$; (vi) chronic renal failure, chronic hepatic dysfunction, and serious heart disease $(n=4)$; and (vii) pregnancy $(n=1$; Fig. 1). Finally, a total of 343 patients were identified after applying all exclusion criteria; among these, $257(75.0 \%)$ had a proximal fracture, $79(23.0 \%)$ had a shaft fracture, and $7(2.0 \%)$ had a distal fracture.

\section{Ethics approval}

This retrospective study was conducted in accordance with the principles outlined in the Declaration of Helsinki and was approved by the Research Ethics Committee of Affiliated Hospital of Zunyi Medical University (reference number: KLL-2020-022). Informed consent was waived by the Research Ethics Committee of Affiliated Hospital of Zunyi Medical University due to the anonymous nature of the data.

\section{Perioperative adverse outcomes}

Perioperative complications, such as lower limb vein thrombus (ICD-10, 180.301), pulmonary embolism (ICD-10, I26), hospital-acquired pneumonia (ICD-10, J12-18), incision disunion (ICD-10, T81.406 or T81.009), bedsores (ICD-10, L89), and transfer to the intensive care unit and death (ICD-10, R99), were defined as adverse perioperative outcomes. The observation period was from admission to discharge.

\section{Variables}

The following various potential influencing factors were investigated: (i) demographic characteristics, including sex, age, weight, chronic diseases, combined injuries, aetiology of fracture, fracture site, postoperative hospital stay, and time to admission (time to admission was graded as 1 (within $24 \mathrm{~h}$ ), 2 (2-3 days), 3 (4-7 days), 4 (8-21 days), or 5 (> 22 days)); (ii) surgery and anaesthesia records, including 
the American Society of Anesthesiologists (ASA) grade, surgeons (eight chief surgeons with at least 20 years of surgical experience (surgeons A, B, C, D, E, F, G, and H) participated in this study), surgery type, anaesthesia methods, postoperative analgesic methods, duration of anaesthesia and surgery, ratio of perioperative blood transfusion, intraoperative blood loss, and intraoperative crystal and colloidal liquid infusion volumes; and (iii) laboratory results at admission, 1 day postoperatively, and before discharge ("before discharge" was defined as the results of the latest blood test, usually within $48 \mathrm{~h}$ before discharge), including ALB, prealbumin (PAb), globulin (GLB), and Hb concentrations, lymphocyte (LYM) and neutrophil (NEUT) counts, neutrophil to lymphocyte ratio (NLR), and PNI. PNI was calculated using the following formula: $10 \times$ ALB $(\mathrm{g} / \mathrm{dl})+0.005 \times \operatorname{LYM}$ count $\left(\text { per } \mathrm{mm}^{3}\right)^{13-15}$ (Table 1). 
Table 1

Comparison between patients with and without adverse outcomes

\begin{tabular}{|c|c|c|c|}
\hline Variables & $\begin{array}{l}\text { No adverse } \\
\text { outcomes } \\
(n=184)\end{array}$ & $\begin{array}{l}\text { Adverse outcomes } \\
(n=159)\end{array}$ & $\begin{array}{l}P \\
\text { values }\end{array}$ \\
\hline Male/female, $n(\%)$ & 88 (47.8)/96 (52.2) & 70 (44.0)/89 (56.0) & 0.481 \\
\hline Left/right femur, $n(\%)$ & $94(51.1) / 90(48.9)$ & 78 (49.1)/81 (50.9) & 0.708 \\
\hline Age, years, mean (range) & $60(43.25-71)$ & $70(60-81)$ & $<0.001$ \\
\hline Weight, kg, mean (range) & $55(50-62)$ & $55(50-60)$ & 0.259 \\
\hline Hypertension, $n(\%)$ & $45(24.5)$ & $74(46.5)$ & $<0.001$ \\
\hline Diabetes, $n(\%)$ & $12(6.5)$ & $18(11.3)$ & 0.117 \\
\hline Combined injuries, $n(\%)$ & $112(43.1)$ & $47(56.6)$ & 0.031 \\
\hline Aetiology, $n(\%)$ & & & 0.968 \\
\hline Sprain or tumble & $142(77.2)$ & $123(77.4)$ & \\
\hline RTA, falls, or assaults & $42(22.8)$ & $36(22.6)$ & \\
\hline Fracture site, $n(\%)$ & & & 0.493 \\
\hline Proximal femoral fracture & $140(76.1)$ & $117(73.6)$ & \\
\hline Femoral shaft fracture & $39(21.2)$ & $40(25.2)$ & \\
\hline Distal femoral fracture & $5(2.7)$ & $2(1.3)$ & \\
\hline Time to admission, $n(\%)$ & & & 0.002 \\
\hline 1: Within $24 \mathrm{~h}$ & $127(69.0)$ & $81(50.9)$ & \\
\hline 2: $2-3$ days & $17(9.2)$ & $24(15.1)$ & \\
\hline 3: 4-7 days & $14(7.6)$ & $19(11.9)$ & \\
\hline 4: 8-21 days & $12(6.5)$ & $26(16.4)$ & \\
\hline $5: \geq 22$ days & $14(7.6)$ & $9(5.7)$ & \\
\hline ASA, $n(\%)$ & & & $<0.001$ \\
\hline$\square$ & $4(2.2)$ & $2(1.3)$ & \\
\hline \multicolumn{4}{|c|}{ Bold text represents confounding factors with $P<0.10$. } \\
\hline \multicolumn{4}{|c|}{$\begin{array}{l}\text { ALB, albumin, } A S A \text {, American Society of Anesthesiologists, } G L B \text {, globulin, } H b \text {, haemoglobin, Intra-; } \\
\text { intraoperative, } L Y M \text {, lymphocyte, } N E U T \text {, neutrophil, } N L R \text {, neutrophil to lymphocyte ratio, } P A b \text {, } \\
\text { Prealbumin, } P C E A \text {, patient-controlled epidural analgesia, } P C I A \text {, patient-controlled intravenous } \\
\text { analgesia, } P N I \text {, prognostic nutritional index, } R T A \text {, road traffic accidents. }\end{array}$} \\
\hline
\end{tabular}




\begin{tabular}{|c|c|c|c|}
\hline Variables & $\begin{array}{l}\text { No adverse } \\
\text { outcomes } \\
(n=184)\end{array}$ & $\begin{array}{l}\text { Adverse outcomes } \\
(n=159)\end{array}$ & $\begin{array}{l}P \\
\text { values }\end{array}$ \\
\hline 口 & $157(85.3)$ & $106(66.7)$ & \\
\hline प & $23(12.5)$ & $50(31.4)$ & \\
\hline 口 & $0(0)$ & $1(0.6)$ & \\
\hline Surgeons, $n(\%)$ & & & 0.161 \\
\hline Surgeon A & $28(15.2)$ & $22(13.8)$ & \\
\hline Surgeon B & $38(20.7)$ & $21(13.2)$ & \\
\hline Surgeon $\mathrm{C}$ & $22(12.0)$ & $18(11.3)$ & \\
\hline Surgeon D & $27(14.7)$ & $22(13.8)$ & \\
\hline Surgeon $\mathrm{E}$ & $31(16.8)$ & $32(20.1)$ & \\
\hline Surgeon F & $13(7.1)$ & $17(10.7)$ & \\
\hline Surgeon G & $18(9.8)$ & $11(6.9)$ & \\
\hline Surgeon $\mathrm{H}$ & $7(3.8)$ & $16(10.1)$ & \\
\hline Operation types, $n(\%)$ & & & 0.014 \\
\hline Hemi/total-hip hip replacement & $73(39.7)$ & $43(27.0)$ & \\
\hline Internal fixation & $111(60.3)$ & $116(73.0)$ & \\
\hline Anaesthesia methods, $n(\%)$ & & & 0.445 \\
\hline General anaesthesia & $47(25.5)$ & $35(22.0)$ & \\
\hline Non-general anaesthesia & $137(74.5)$ & $124(78.0)$ & \\
\hline Postoperative analgesic methods, $n$ (\%) & & & 0.315 \\
\hline No postoperative analgesia & $9(4.9)$ & $4(2.5)$ & \\
\hline PCIA & $123(66.8)$ & $101(63.5)$ & \\
\hline PCEA & $52(28.3)$ & $54(34.0)$ & \\
\hline Duration of anaesthesia, min, mean (range) & $180(140-225)$ & $180(149-230)$ & 0.323 \\
\hline
\end{tabular}

Bold text represents confounding factors with $P<0.10$.

$A L B$, albumin, $A S A$, American Society of Anesthesiologists, $G L B$, globulin, $H b$, haemoglobin, Intraintraoperative, $L Y M$, lymphocyte, $N E U T$, neutrophil, $N L R$, neutrophil to lymphocyte ratio, $P A b$, Prealbumin, $P C E A$, patient-controlled epidural analgesia, $P C I A$, patient-controlled intravenous analgesia, $P N I$, prognostic nutritional index, RTA, road traffic accidents. 


\begin{tabular}{|c|c|c|c|}
\hline Variables & $\begin{array}{l}\text { No adverse } \\
\text { outcomes } \\
(n=184)\end{array}$ & $\begin{array}{l}\text { Adverse outcomes } \\
(n=159)\end{array}$ & $\begin{array}{l}P \\
\text { values }\end{array}$ \\
\hline Duration of surgery, min, mean (range) & $111(80-153.5)$ & $110(80-155)$ & 0.914 \\
\hline Blood transfusion, $n(\%)$ & $61(33.2)$ & $87(54.7)$ & $<0.001$ \\
\hline Intra-blood loss, $\times 10^{2} \mathrm{ml}$, mean (range) & $2(1-3)$ & $2(1-3)$ & 0.052 \\
\hline Intra-crystal liquids, $\times 10^{2} \mathrm{ml}$, mean (range) & $8.5(6-11)$ & $7.5(6-11)$ & 0.662 \\
\hline $\begin{array}{l}\text { Intra-colloidal liquids, } \times 10^{2} \mathrm{ml} \text {, mean } \\
\text { (range) }\end{array}$ & $5(5-7.5)$ & $5(5-10)$ & 0.983 \\
\hline $\begin{array}{l}\text { Postoperative hospital stay, days, mean } \\
\text { (range) }\end{array}$ & $6(4-7)$ & $7(5-9)$ & $<0.001$ \\
\hline \multicolumn{4}{|l|}{ Blood test at admission } \\
\hline$A L B, g / l$, mean $\pm S D$ & $38 \pm 3.91$ & $34.48 \pm 4.34$ & $<0.001$ \\
\hline PAb, mg/l, mean (range) & $191(154-229.5)$ & $158(126-194)$ & $<0.001$ \\
\hline $\mathrm{Hb}, \mathrm{g} / \mathrm{l}$, mean $\pm \mathrm{SD}$ & $120.02 \pm 18.96$ & $108.24 \pm 20.99$ & $<0.001$ \\
\hline GLB, g/l, mean (range) & $\begin{array}{l}27.15(23.35- \\
29.95)\end{array}$ & $\begin{array}{l}27.70(24.90- \\
30.80)\end{array}$ & 0.110 \\
\hline LYM count, $\times 10^{9} /$, mean (range) & $1.30(0.98-1.68)$ & $1.12(0.87-1.50)$ & 0.004 \\
\hline NEUT count, $\times 10^{9} / I$, mean (range) & $5.52(4.31-7.16)$ & $4.97(3.92-6.43)$ & 0.035 \\
\hline NLR, mean (range) & $4.21(2.96-6.09)$ & $4.71(3.27-7.20)$ & 0.150 \\
\hline $\mathrm{PNI}$, mean $\pm \mathrm{SD}$ & $44.54 \pm 5.05$ & $39.30 \pm 4.99$ & $<0.001$ \\
\hline \multicolumn{4}{|l|}{ Blood test at 1 day postoperatively } \\
\hline ALB, g/l, mean (range) & $29.2(27.2-32.58)$ & $27.4(24.6-29.3)$ & $<0.001$ \\
\hline PAb, mg/l, mean (range) & $104(75-147)$ & $86(66-114)$ & $<0.001$ \\
\hline $\mathrm{Hb}, \mathrm{g} / \mathrm{l}$, mean (range) & $91(78-99.75)$ & $83(72-93)$ & $<0.001$ \\
\hline GLB, g/l, mean (range) & $\begin{array}{l}22.70(19.20- \\
25.28)\end{array}$ & $\begin{array}{l}21.40(19.10- \\
24.00)\end{array}$ & 0.045 \\
\hline
\end{tabular}

Bold text represents confounding factors with $P<0.10$.

$A L B$, albumin, $A S A$, American Society of Anesthesiologists, $G L B$, globulin, $H b$, haemoglobin, Intraintraoperative, $L Y M$, lymphocyte, $N E U T$, neutrophil, $N L R$, neutrophil to lymphocyte ratio, $P A b$, Prealbumin, $P C E A$, patient-controlled epidural analgesia, $P C I A$, patient-controlled intravenous analgesia, $P N I$, prognostic nutritional index, RTA, road traffic accidents. 


\begin{tabular}{|c|c|c|c|}
\hline Variables & $\begin{array}{l}\text { No adverse } \\
\text { outcomes } \\
(n=184)\end{array}$ & $\begin{array}{l}\text { Adverse outcomes } \\
(n=159)\end{array}$ & $\begin{array}{l}P \\
\text { values }\end{array}$ \\
\hline LYM count, $\times 10^{9} /$, mean (range) & $0.84(0.67-1.14)$ & $0.73(0.53-0.97)$ & 0.001 \\
\hline NEUT count, $\times 10^{9} / \mathrm{L}$, mean (range) & $7.81(5.92-10.01)$ & $8.40(6.60-11.41)$ & 0.026 \\
\hline NLR, mean (range) & $9.06(5.86-13.49)$ & $\begin{array}{l}10.20(8.07- \\
14.86)\end{array}$ & 0.001 \\
\hline PNI, mean (range) & $\begin{array}{l}34.15(31.55- \\
37.61)\end{array}$ & $\begin{array}{l}31.70(29.05- \\
34.20)\end{array}$ & $<0.001$ \\
\hline \multicolumn{4}{|l|}{ Blood test before discharge } \\
\hline ALB, $g / l$, mean $\pm S D$ & $32.75 \pm 3.44$ & $32.32 \pm 4.14$ & 0.293 \\
\hline PAb, mg/l, mean (range) & $112.5(86.25-160)$ & $104(80-141)$ & 0.028 \\
\hline $\mathrm{Hb}, \mathrm{g} / \mathrm{l}$, mean (range) & $93(83-102.75)$ & $91(82-100)$ & 0.129 \\
\hline GLB, g/l, mean (range) & $\begin{array}{l}24.50(21.73- \\
27.30)\end{array}$ & $\begin{array}{l}24.20(22.10- \\
27.90)\end{array}$ & 0.614 \\
\hline LYM count, $\times 10^{9} / \mathrm{L}$, mean (range) & $1.04(0.81-1.41)$ & $1.04(0.71-1.33)$ & 0.140 \\
\hline NEUT count, $\times 10^{9} / L$, mean (range) & $5.76(4.36-7.30)$ & $5.31(4.17-7.21)$ & 0.431 \\
\hline NLR, mean (range) & $5.29(3.72-8.08)$ & $5.56(3.78-8.29)$ & 0.440 \\
\hline PNI, mean (range) & $\begin{array}{l}37.83(34.75- \\
41.05)\end{array}$ & $\begin{array}{l}37.05(33.85- \\
41.60)\end{array}$ & 0.152 \\
\hline \multicolumn{4}{|c|}{ Bold text represents confounding factors with $P<0.10$. } \\
\hline \multicolumn{4}{|c|}{$\begin{array}{l}A L B \text {, albumin, } A S A \text {, American Society of Anesthesiologists, } G L B \text {, globulin, } H b \text {, haemoglobin, Intra-, } \\
\text { intraoperative, } L Y M \text {, lymphocyte, } N E U T \text {, neutrophil, } N L R \text {, neutrophil to lymphocyte ratio, } P A b \text {, } \\
\text { Prealbumin, } P C E A \text {, patient-controlled epidural analgesia, } P C I A \text {, patient-controlled intravenous } \\
\text { analgesia, } P N I \text {, prognostic nutritional index, } R T A \text {, road traffic accidents. }\end{array}$} \\
\hline
\end{tabular}

\section{Statistical analysis}

Continuous data are presented as mean with standard deviation or median (interquartile range) according to statistical distribution (assumption of normality was assessed using the Kolmogorov-Smirnov test). Categorical parameters are presented as frequencies and associated percentages. The Student's $t$-test was used to analyse normally distributed continuous variables, whereas the Mann-Whitney Utest was utilised to examine non-normally distributed continuous variables and ordinal variables (ASA grade, surgical grades, and time to admission). The chi-square or Fisher's exact test was employed to analyse categorical variables. In these analyses, variables with unadjusted $p<0.10$ were identified as confounding factors and were included in multivariate regression analyses to 
determine independent predictors of adverse perioperative outcomes. The results are expressed as OR and $95 \% \mathrm{Cl}$. The discriminatory ability of the independent predictors was assessed using the receiver operating characteristic (ROC) curve analysis. Optimal cut-off values were obtained using the Youden index, and DeLong's test was used to compare the area under the curve (AUC) with MedCalc statistical software version 19.3.1 (MedCalc Software Ltd., Ostend, Belgium). A $p$ value of $<0.05$ was considered statistically significant. All tests were two-sided. All statistical analyses were conducted using Statistical Package for Social Sciences version 17.0 (SPSS Statistics for Windows, Chicago, USA).

\section{Results}

\section{Patients}

A total of 159 (46.4\%) patients who underwent surgery for femoral fractures experienced adverse perioperative outcomes. Among these, 123 (35.9\%) had lower limb vein thrombus, 68 (19.8\%) had hospital-acquired pneumonia, $6(1.7 \%)$ were transferred to the postoperative intensive care unit, $4(1.2 \%)$ had pulmonary embolism, $3(0.9 \%)$ died during hospitalisation, $2(0.6 \%)$ had incision disunion, and 7 $(2.0 \%)$ had other adverse outcomes, including renal and liver function impairment, acute heart failure, acute cerebral infarction, and stress gastroenteritis (Table 1).

\section{Confounding and independent factors}

The following factors were associated with adverse outcomes: age; hypertension; combined injuries; time to admission; ASA classification; surgery type; ratio of perioperative blood transfusion; intraoperative blood loss; postoperative hospital stay; admission values of ALB, PAb, Hb, LYM count, NEUT count, and PNl; 1-day postoperative values of ALB, PAb, Hb, GLB, LYM count, NEUT count, NLR, and PNl; and PAb value before discharge (all $P$ values $<0.10 ;$ Table 1 ). All the aforementioned confounding factors, except for ALB concentrations (which showed collinearity with $\mathrm{PNI}$ ), were included in the multivariate regression analyses to determine the independent factors associated with adverse perioperative outcomes. The PNI at admission (odds ratio [OR]: 0.850, 95\% confidence interval [Cl]: 0.776-0.931, $P<0.001$ ), age (OR: $1.041,95 \% \mathrm{Cl}: 1.016-1.066, P=0.001)$, postoperative hospital stay (OR: $1.132,95 \% \mathrm{Cl}: 1.016-1.263, P=$ 0.025), time to admission (OR: $1.343,95 \% \mathrm{Cl}: 1.056-1.708, P=0.016$ ), hypertension (OR: $2.091,95 \% \mathrm{Cl}$ : 1.116-3.916, $P=0.021$ ), combined injures (OR: $2.836,95 \% \mathrm{Cl}: 1.340-6.003, P=0.006$ ), and surgery type (OR: $4.625,95 \% \mathrm{Cl}: 2.283-9.367, P<0.001$ ) were identified as independent factors for perioperative adverse outcomes (Table 2). 
Table 2

Multivariate regression analyses of confounding factors

\begin{tabular}{|c|c|c|}
\hline Confounding factors & OR $(95 \% \mathrm{Cl})$ & $P$ values \\
\hline PNI at admission (per 1) & $0.850(0.776,0.931)$ & $<0.001$ \\
\hline Age (per 1 year) & $1.041(1.016,1.066)$ & 0.001 \\
\hline Postoperative hospital stay (per 1 day) & $1.132(1.016,1.263)$ & 0.025 \\
\hline Time to admission (per 1 ) & $1.343(1.056,1.708)$ & 0.016 \\
\hline Hypertension (ref: no) & $2.091(1.116,3.916)$ & 0.021 \\
\hline Combined injuries (ref: no) & $2.836(1.340,6.003)$ & 0.006 \\
\hline Operation types (ref: hip replacement) & $4.625(2.283,9.367)$ & $<0.001$ \\
\hline 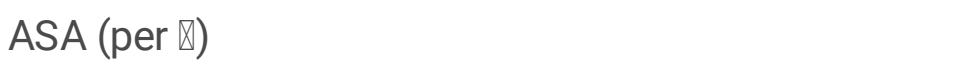 & $1.411(0.718,2.773)$ & 0.317 \\
\hline Blood transfusion (ref: no) & $1.040(0.500,2.163)$ & 0.916 \\
\hline Intraoperative blood loss (per $1 \times 10^{2} \mathrm{ml}$ ) & $1.042(0.878,1.238)$ & 0.638 \\
\hline PAb at admission (per $1 \mathrm{mg} / \mathrm{l}$ ) & $1.000(0.993,1.008)$ & 0.950 \\
\hline $\mathrm{Hb}$ at admission (per $1 \mathrm{~g} / \mathrm{l}$ ) & $1.015(0.990,1.040)$ & 0.244 \\
\hline LYM count at admission (per $1 \times 10^{9} / \mathrm{I}$ ) & $1.520(0.677,3.411)$ & 0.310 \\
\hline NETU count at admission (per $1 \times 10^{9} / \mathrm{I}$ ) & $1.015(0.892,1.155)$ & 0.822 \\
\hline PAb at 1 day postoperatively (per $1 \mathrm{mg} / \mathrm{l}$ ) & $1.009(0.995,1.023)$ & 0.191 \\
\hline $\mathrm{Hb}$ at 1 day postoperatively (per $1 \mathrm{~g} / \mathrm{l}$ ) & $0.989(0.959,1.019)$ & 0.460 \\
\hline GLB at 1 day postoperatively (per $1 \mathrm{~g} / \mathrm{l}$ ) & $0.990(0.915,1.071)$ & 0.804 \\
\hline LYM count at 1 day postoperatively (per $1 \times 10^{9} / \mathrm{I}$ ) & $1.016(0.249,4.143)$ & 0.982 \\
\hline NETU count at 1 day postoperatively (per $\left.1 \times 10^{9} / \mathrm{I}\right)$ & $1.055(0.936,1.189)$ & 0.377 \\
\hline NLR at 1 day postoperatively (per 1 ) & $0.991(0.922,1.064)$ & 0.797 \\
\hline $\mathrm{PNI}$ at 1 day postoperatively (per 1 ) & $0.925(0.822,1.040)$ & 0.193 \\
\hline PAb before discharge (per $1 \mathrm{mg} / \mathrm{l}$ ) & $0.997(0.987,1.008)$ & 0.630 \\
\hline
\end{tabular}

The ALB concentration was not included in the model and showed significant collinearity with PNI. Bold fonts represent independent factors with $P<0.05$.

$A L B$, albumin, $A S A$, American Society of Anesthesiologists, $G L B$, globulin, $H b$, haemoglobin, $L Y M$, lymphocyte, NEUT, neutrophil, $N L R$, neutrophil to lymphocyte ratio, $P A b$, Prealbumin, $P N I$, prognostic nutritional index, $O R$, odds ratio. 


\section{AUC and optimal cut-off values of the independent factors and ALB}

Predictive values of the independent factors ( $\mathrm{PNI}$ at admission, age, postoperative hospital stay, and time to admission) were assessed using the ROC curve analysis. Based on the AUC (PNI at admission: 0.772, $95 \% \mathrm{Cl}: 0.723-0.821, P<0.001$; age: $0.678,95 \% \mathrm{Cl}: 0.622-0.734, P<0.001$; postoperative hospital stay: $0.608,95 \% \mathrm{Cl}: 0.548-0.668, P=0.001$; time to admission: $0.585,95 \% \mathrm{Cl}: 0.525-0.646, P=0.006)$, the PNI at admission had the most optimal discrimination ability and was superior to other independent factors (age vs. PNI at admission, $P=0.002$; postoperative hospital stay vs. $\mathrm{PNI}$ at admission, $P<0.001$; time to admission vs. $\mathrm{PNI}$ at admission, $P<0.001)$. As the ALB concentration is a primary measure included in the PNI, there might be a relationship between the ALB and adverse outcomes. However, the PNI at admission was a better predictor $(P=0.038)$ than the ALB concentration at admission $(0.736,95 \% \mathrm{Cl}$ : $0.683-0.790, P<0.001)$. The optimal cut-off values of PNI at admission, age, postoperative hospital stay, time to admission, and ALB concentration at admission were 42.425, 55.5 years, 6.5 days, 2 days, and $36.35 \mathrm{~g} / \mathrm{l}$, respectively (Table 3 ).

Table 3

Comparison of the AUC for independent factors and ALB

\begin{tabular}{|llllll|}
\hline & AUC $(95 \% \mathrm{Cl})$ & $P$ & Youden max & Threshold & $P^{*}$ \\
\hline PNI at admission & $0.772(0.723,0.821)$ & $<0.001$ & 0.425 & 42.425 & \\
\hline Age & $0.678(0.622,0.734)$ & $<0.001$ & 0.250 & 55.5 years & 0.002 \\
\hline Postoperative hospital stay & $0.608(0.548,0.668)$ & 0.001 & 0.196 & 6.5 days & $<0.001$ \\
\hline Time to admission & $0.585(0.525,0.646)$ & 0.006 & 0.181 & 2 days & $<0.001$ \\
\hline ALB at admission & $0.736(0.683,0.790)$ & $<0.001$ & 0.384 & $36.35 \mathrm{~g} / \mathrm{l}$ & 0.038 \\
\hline Bold fonts represent statistical significance, $P<0.05 ; P^{*}<0.05$ vs. PNI. & & \\
\hline ALB, albumin, $A U C$, area under the curve, $P N I$, prognostic nutritional index,. & & \\
\hline
\end{tabular}

\section{Discussion}

This study revealed that the PNI at admission, age, postoperative hospital stay, time to admission, hypertension, combined injuries, and surgery type were independent factors for adverse perioperative outcomes in patients with femoral fractures, and the PNI at admission was likely a better independent predictor than the others. Our findings suggested that nutritional assessment and appropriate intervention strategies at admission are necessary for patients with femoral fractures.

Accumulating evidence indicates that approximately $20-40 \%$ of the patients show an acute, prolonged, and profound decrease in postoperative serum ALB concentrations. ${ }^{16}$ This study showed that the mean 
ALB concentration in patients with or without adverse outcomes 1 day postoperatively were both $<30 \mathrm{~g} / \mathrm{l}$. This indicates that there may be a non-negligible nutrition risk in patients with femoral fractures during the perioperative period. However, these patients were administered parenteral nutrition interventions as routine care when the ALB concentration was $<30 \mathrm{~g} / \mathrm{l}$.

The PNI is a pre-treatment nutritional risk stratification tool that utilises the combined effects of hypoalbuminemia and lymphocytopenia to assess the immunologic and nutritional aspects of patients undergoing surgery. ${ }^{12,16,17}$ Lower serum ALB concentrations and total lymphocyte counts are important risk factors for predicting the 1-year mortality of elderly patients with intertrochanteric fractures. ${ }^{18}$ Consistently, in this study, the multivariate regression analysis showed that the nutritional status at admission, not the postoperative nutritional status, was negatively correlated with adverse perioperative outcomes in patients with femoral fractures. Although age $(P=0.001)$, time to admission $(P=0.016)$, hypertension $(P=0.021)$, combined injures $(P=0.006)$, and surgery type $(P<0.001)$ were also independent factors, they were not necessarily controllable. Additionally, it is considered that there is an interactive relationship and a reciprocal causation between postoperative hospital stay $(P=0.025)$ and perioperative adverse outcomes. Notably, the ROC curve analysis showed that the PNI at admission might provide better predictive value than other independent factors in this study, including age and time to admission. As the ALB concentration is a primary measure included in the PNI (in addition to lymphocyte concentration), the ALB concentration at admission might have been associated with adverse outcomes as well. Nevertheless, the PNI at admission was superior to the ALB at admission in predicting adverse perioperative outcomes in patients with femoral fractures $(P=0.038)$. Collectively, the $\mathrm{PNI}$ at admission was a better and more effective predictor of adverse perioperative outcomes. Based on the high correlation between nutrition and adverse perioperative outcomes, this study suggested that all patients with femoral fractures should undergo a nutritional assessment and nutritional intervention at admission, but not in the presence of malnutrition or hypoalbuminemia, or postoperatively.

Hypertension is closely related to vascular endothelial cell injury and is often accompanied by dyslipidaemia, and both vascular endothelial cell injury and dyslipidaemia are associated with the formation of venous thrombus. In this study, we observed that thrombus accounted for $77.4 \%(123 / 159)$ of adverse perioperative outcomes. This finding suggests that hypertension is an independent risk factor for adverse perioperative outcomes.

The type of surgery was classified into only two primary categories in this study: hemi/total-hip replacement and internal fixation (mainly consisting of intramedullary nailing, cannulated-screw, and plate-screw internal fixation). The former is primarily performed in elderly patients with proximal femoral fractures, and the latter is performed commonly in younger or non-hip fracture patients. There are differences in incision, surgery duration, degree of ache, blood loss, and hospital stay among patients treated by different surgical methods. In this study, we comprehensively evaluated the surgery-related factors, and the results indicated that the number of patients with femoral fractures who underwent internal fixation was 4.6 times the number of patients with femoral fractures who underwent hemi/totalhip replacement. The possible reasons were more severe pain, bleeding, inflammation, activity limitation, 
and a longer bedridden period in the internal fixation-treated patients than in the hemi/total-hip replacement patients.

The NLR is considered a prognostic factor for outcomes and survival in cardiology, oncology, and gastrointestinal surgery. ${ }^{19}$ It is also a risk factor for postoperative mortality and cardiovascular complications in elderly patients undergoing surgery for hip fracture repair. ${ }^{19,20}$ The NEUT count, an effective and cheap inflammatory marker, is widely applied in clinical practice to guide diagnosis and therapy. In this report, we selected the NLR and NEUT count to represent perioperative inflammatory reaction. However, neither the NLR nor the NEUT count affected the adverse perioperative outcomes in patients with femoral fractures. This result may have been due to the administration of perioperative antibiotic prophylaxis in all patients, and because perioperative inflammation gets more attention than nutrition from surgeons and anaesthetists in China.

There are several limitations of this study. First, this was a single-centre study. Second, body mass index (BMI) was not evaluated in this study. $\mathrm{BMI}$ is an indicator for the assessment of nutritional status and a good predictor of morbidity and mortality; ${ }^{21}$ however, the height values were not documented in this study, mainly because patients with femoral fractures were unable to stand up to provide an accurate height measurement. Third, the lipid profile was not measured in most of the enrolled patients. Further studies are needed to evaluate the lipid profile (total cholesterol, triglycerides, and lipoprotein

concentrations) as the lipid profile is associated with the risk of venous thrombus. ${ }^{22,23}$ Finally, we did not observe the long-term complications and mortality.

\section{Conclusions}

In conclusion, this study showed that age, hypertension, combined injuries, and internal fixation were independent risk factors for adverse perioperative outcomes in patients with femoral fractures. Early admission to hospital for treatment and shorter duration of postoperative hospital stay were associated with a decrease in the incidence of adverse perioperative outcomes. Most importantly, our findings suggested that all patients with femoral fractures require a nutritional assessment and nutrition intervention on admission, and that the PNI value at admission may be a good nutritional assessment indicator.

\section{Abbreviations}

prognostic nutritional index (PNI)

area under the curve (AUC)

human serum albumin (ALB)

International Classification of Diseases, Tenth Revision (ICD-10) 
American Society of Anesthesiologists (ASA)

prealbumin (PAb)

globulin (GLB)

lymphocyte (LYM)

neutrophil (NEUT)

neutrophil to lymphocyte ratio (NLR)

odds ratio (OR)

confidence interval (Cl)

body mass index (BMI)

\section{Declarations}

\section{Ethics approval and consent to participate}

This retrospective study was conducted in accordance with the principles outlined in the Declaration of Helsinki and was approved by the Research Ethics Committee of Affiliated Hospital of Zunyi Medical University (reference number: KLL-2020-022). Informed consent was waived by the Research Ethics Committee of Affiliated Hospital of Zunyi Medical University due to the anonymous nature of the data.

\section{Consent for publication}

Not applicable.

\section{Availability of data and materials}

The datasets analysed during the current study are available from the corresponding author on reasonable request.

\section{Acknowledgments}

We are grateful for the help from other staff, postgraduates, and undergraduates who were involved in data collection in this study.

\section{Competing interests}

The authors declare no conflict of interest.

\section{Author contributions}


$\mathrm{MH}$ and QF designed and oversaw the study, prepared the statistical analysis plan, and wrote the manuscript. $Y Z, D L, X L, S X, J P$, and ZZ contributed to the design. ZZ revised the paper and had primary responsibility for the final content. All authors read and approved the final manuscript.

\section{Funding}

This study received no specific grant from any funding agency, commercial or not-for-profit sectors.

\section{References}

1. Melton LJ 3rd. Hip fractures: a worldwide problem today and tomorrow. Bone. 1993;14 Suppl 1:S1S8. doi: 10.1016/8756-3282(93)90341-7.

2. Hughes MS, Kazmier P, Burd TA, Anglen J, Stoker AM, Kuroki K, Carson WL, Cook JL. Enhanced fracture and soft-tissue healing by means of anabolic dietary supplementation. J Bone Joint Surg Am. 2006;88:2386-2394. doi: 10.2106/JBJS.F.00507.

3. Haentjens P, Autier P, Barette M, Boonen S; Belgian Hip Fracture Study Group. Costs of care after hospital discharge among women with a femoral neck fracture. Clin Orthop Relat Res. 2003;414:250-258. doi: 10.1097/01.blo.0000079262.91782.04.

4. Amarilla-Donoso FJ, Lopez-Espuela F, Roncero-Martin R. Quality of life in elderly people after a hip fracture: a prospective study. Health Qual Life Outcomes. 2020;18:71. doi: 10.1186/s12955-02001314-2.

5. Giancola R, Marchesi LP, Lettera MG, Antonini G. A complex proximal femoral fracture. Injury. 2016;47 Suppl 4:S121-S123. doi: 10.1016/j.injury.2016.07.049.

6. Lutnick E, Kang J, Freccero DM. Surgical treatment of femoral neck fractures: a brief review. Geriatrics (Basel). 2020;5:22. doi: 10.3390/geriatrics5020022.

7. Cummings SR, Melton LJ. Epidemiology and outcomes of osteoporotic fractures. Lancet. 2002;359:1761-1767. doi: 10.1016/S0140-6736(02)08657-9.

8. Gosling T, Krettek C. Femoral shaft fractures. Unfallchirurg. 2019;122:59-75. doi: 10.1007/s00113018-0591-7.

9. Young EY, Stans AA. Distal femoral physeal fractures. J Knee Surg. 2018;31:486-489. doi: 10.1055/s-0038-1627465.

10. Meesters DM, Wijnands KAP, Brink PRG, Poeze M. Malnutrition and fracture healing: are specific deficiencies in amino acids important in nonunion development? Nutrients. 2018;10:1597. doi: 10.3390/nu10111597.

11. Invernizzi M, de Sire A, D'Andrea F, Carrera D, Renò F, Migliaccio S, Iolascon G, Cisari C. Effects of essential amino acid supplementation and rehabilitation on functioning in hip fracture patients: a pilot randomised controlled trial. Aging Clin Exp Res. 2019;31: 1517-1524. doi: 10.1007/s40520018-1090-y. 
12. Buzby GP, Mullen JL, Matthews DC, Hobbs CL, Rosato EF. Prognostic nutritional index in gastrointestinal surgery. Am J Surg. 1980;139:160-167. doi: 10.1016/0002-9610(80)90246-9.

13. Caputo F, Dadduzio V, Tovoli F, Bertolini G, Cabibbo G, Cerma K et al. The role of PNI to predict survival in advanced hepatocellular carcinoma treated with Sorafenib. PLoS One. 2020;15:e0232449. doi: 10.1371/journal.pone.0232449.

14. Ren H, Wu L, Hu W, Ye X, Yu B. Prognostic value of the C-reactive protein/prognostic nutritional index ratio after hip fracture surgery in the elderly population. Oncotarget. 2017;8:61365-61372. doi: 10.18632/oncotarget.18135.

15. Cadwell JB, Afonso AM, Shahrokni A. Prognostic nutritional index (PNI), independent of frailty is associated with six-month postoperative mortality. J Geriatr Oncol. 2020;11:880-884. doi: 10.1016/j.jgo.2020.03.013.

16. Li X, Chen J. Is the prognostic nutritional index (PNI) a useful predictive marker for postoperative complications after lung surgery? J Thorac Dis. 2019;11:S334-S336. doi: 10.21037/jtd.2018.12.124.

17. Onodera T, Goseki N, Kosaki G. Prognostic nutritional index in gastrointestinal surgery of malnourished cancer patients. Nihon Geka Gakkai Zasshi. 1984;85:1001-1005.

18. Lu J, Chen YY, Zhang L, Li YG, Wang C. Laboratory nutritional parameters predict one-year mortality in elderly patients with intertrochanteric fracture. Asia Pac J Clin Nutr. 2016; 25:457-463. doi: 10.6133/apjen.092015.04.

19. Forget P, Moreau N, Engel H, Cornu O, Boland B, De Kock M, Yombi JC. The neutrophil-to-lymphocyte ratio (NLR) after surgery for hip fracture (HF). Arch Gerontol Geriatr. 2015;60:366-371. doi: 10.1016/j.archger.2014.11.008.

20. Forget P, Dillien P, Engel H, Cornu O, De Kock M, Yombi JC. Use of the neutrophil-to-lymphocyte ratio as a component of a score to predict postoperative mortality after surgery for hip fracture in elderly subjects. BMC Res Notes. 2016;9:284. doi: 10.1186/s13104-016-2089-0.

21. Miller MD, Thomas JM, Cameron ID, Chen JS, Sambrook PN, March LM, Cumming RG, Lord SR. BMI: a simple, rapid and clinically meaningful index of under-nutrition in the oldest old? Br J Nutr. 2009;101:1300-1305. doi: 10.1017/S0007114508076289.

22. Garcia-Raso A, Ene GS, Sillero PL. Lipid profile is associated with risk of thrombotic complications. Thromb Haemost. 2013;110:390-392. doi: 10.1160/TH12-12-0896.

23. Garcia-Raso A, Ene GS, Sillero PL. Alterations of lipid profile are a risk factor for venous thromboembolism and thrombotic complications. Eur J Lipid Sci Tech. 2014;116:514-52. doi: 10.1002/ejlt.201300414.

\section{Figures}


Inclusion criteria $(n=446)$ :

Admission for femur fracture

Underwent surgery for a single femur fracture during hospitalization

Operation data from Jan 2018 to Dec 2018

\begin{tabular}{|c|c|}
\hline $\mathbf{E}$ & $\begin{array}{l}\text { clusion criteria }(n=103) \text { : } \\
\text { Reoperation or multi-location operations }(n=44) \\
\text { Incomplete data }(n=27) \\
\text { Tuberculosis }(n=13) \\
\text { Tumors }(n=6) \\
\text { Younger than } 18 \text { years }(n=4) \\
\text { Thromboembolism history }(n=4) \\
\text { Severe nephropathy }(n=3) \\
\text { Serious heart disease }(n=1) \\
\text { Pregnancy }(n=1)\end{array}$ \\
\hline \multirow{2}{*}{\multicolumn{2}{|c|}{ No perioperative adverse outcomes $(n=184,53.6 \%)$}} \\
\hline & \\
\hline \multicolumn{2}{|c|}{$\begin{array}{l}\text { Perioperative adverse outcomes }(\mathrm{n}=159,46.4 \%) \\
\text { Lower limb vein thrombus }(\mathrm{n}=123,35.9 \%) \\
\text { Hospital-acquired pneumonia }(\mathrm{n}=68,19.8 \%) \\
\text { Transfer to postoperative } \mathrm{ICU}(\mathrm{n}=6,1.7 \%) \\
\text { Pulmonary embolism }(\mathrm{n}=4,1.2 \%) \\
\text { Death }(\mathrm{n}=3,0.9 \%) \\
\text { Operative incision disunion }(\mathrm{n}=\mathbf{2 , 0 . 6 \% )} \\
\text { Renal function impairment }(\mathrm{n}=\mathbf{2 , 0 . 6 \% )} \\
\text { Liver function impairment }(\mathrm{n}=\mathbf{2 , 0 . 6 \% )} \\
\text { Acute heart failure }(\mathrm{n}=1,0.3 \%) \\
\text { Acute cerebral infarction }(\mathrm{n}=1,0.3 \%) \\
\text { Stress gastroenteritis }(\mathrm{n}=1,0.3 \%) \\
\text { Bedsores }(\mathrm{n}=0)\end{array}$} \\
\hline
\end{tabular}

Figure 1

Flow chart of patient inclusion. ICU intensive care unit. 